\title{
Comparison of estimation techniques for vibro-acoustic transfer path analysis
}

\author{
Paulo Eduardo França Padilha ${ }^{\mathrm{a}, *}$ and José Roberto de França Arruda ${ }^{\mathrm{b}}$ \\ ${ }^{a}$ General Motors do Brasil Ltda., Campo de Provas da Cruz Alta, Indaiatuba, SP, Brazil \\ ${ }^{\mathrm{b}}$ Universidade Estadual de Campinas, Depatamento de Mecânica Computacional, Barão Geraldo, Campinas, SP, \\ Brazil
}

\begin{abstract}
Vibro-acoustic Transfer Path Analysis (TPA) is a tool to evaluate the contribution of different energy propagation paths between a source and a receiver, linked to each other by a number of connections. TPA is typically used to quantify and rank the relative importance of these paths in a given frequency band, determining the most significant one to the receiver. Basically, two quantities have to be determined for TPA: the operational forces at each transfer path and the Frequency Response Functions (FRF) of these paths. The FRF are obtained either experimentally or analytically, and the influence of the mechanical impedance of the source can be taken into account or not. The operational forces can be directly obtained from measurements using force transducers or indirectly estimated from auxiliary response measurements. Two methods to obtain the operational forces indirectly - the Complex Stiffness Method (CSM) and the Matrix Inversion Method (MIM) - associated with two possible configurations to determine the FRF - including and excluding the source impedance - are presented and discussed in this paper. The effect of weak and strong coupling among the paths is also commented considering the techniques previously presented. The main conclusion is that, with the source removed, CSM gives more accurate results. On the other hand, with the source present, MIM is preferable. In the latter case, CSM should be used only if there is a high impedance mismatch between the source and the receiver. Both methods are not affected by a higher or lower degree of coupling among the transfer paths.
\end{abstract}

Keywords: Vibro-acoustics, transfer, path, structure-borne, noise

\section{Introduction}

Vibro-acoustic Transfer Path Analysis (TPA) is a tool to evaluate the contribution of different energy propagation paths between a source and a receiver, linked to each other by a number of connections. TPA is typically used to rank the relative importance of the vibro-acoustic paths in a frequency band and to determine the most significant path to a given receiver location. Since the path is identified and quantified, techniques of noise and vibration control can be efficiently applied to minimize the overall response level. It should be noted that contributions of each path are vector quantities, that is, their magnitudes are not arithmetically summed to obtain the total level since destructive interference can occur at certain frequencies.

A single source, implying in total coherence among the noise paths, is the simplest case of TPA. A classical example of this situation is the analysis of the interior noise of an automobile caused by the engine operation during the normal usage.

Basically, two quantities have to be determined during the analysis. The operational forces at each transfer path and Frequency Response Functions (FRF) through these paths. The FRF are obtained either experimentally or analytically and the influence of the mechanical impedance of the source can be taken into account or not. The operational forces can be directly obtained from measurements using force transducers or estimated through

${ }^{*}$ Corresponding author: Estrada General Motors s/n, 13347-500, Indaiatuba, SP, Brazil. Tel.: +55 193894 9144; Fax: +55 19 3894 9170; E-mail: paulo.padilha@gm.com. 
the measurement of auxiliary parameters, using indirect techniques. The methods to obtain these quantities and consequently the transfer paths will be presented and discussed in the present work.

The determination of the operational forces directly is not always feasible in practical problems because it requires the placing of force transducers between the source and the attachment points to the receiver structures. At these points, there are usually vibration isolators or engine mounts and the local stiffness can be significantly affected by the presence of the transducers, leading to poor results. In many cases, operational conditions and access limitations can also difficult or even impede the insertion of measuring sensors at these positions. Furthermore, multiple directions are frequently considered for each point (usually the three main translations are considered and rotations neglected) and the transducer must be able to measure them simultaneously.

In TPA, the total receiver response (acceleration or sound pressure) is considered as a superposition of individual contributions of each transfer path. Each individual contribution is calculated as the product of the FRF by the operational force applied to that path. So, the determination of the FRF and the applied operational force is a necessary condition to assess each noise path contribution.

Mathematically:

$$
r_{i}(\omega)=\sum_{j=1}^{n} H_{i j}(\omega) \cdot f_{j}(\omega)
$$

where:

$r_{i}(\omega)$ is the receiver operational response at point $i$ as a function of frequency;

$H_{i j}(\omega)$ is the FRF between the point $i$ and the input signal applied to path $j$;

$f_{j}(\omega)$ is the operational force applied to path $j$.

\section{Methods to estimate the operational forces}

Two indirect methods to determine the operational forces will be studied. The first one is the so-called Complex Stiffness Method (CSM). Typically, this method is applied to problems of structure-borne noise propagation from a source (an engine, for example) connected via resilient mounts to the receiver structure. Each excitation direction on each mount is considered a noise transfer path. The technique consists in determining displacements in each direction, in operational conditions, on both sides of each mount. After obtaining (normally by test) the mount dynamic stiffness for each direction, the operational forces can be calculated multiplying the mount stiffness by the relative displacement of the mount on each direction.

So, for a given path $j$, i.e., for a given mount and direction, the following equation can be written:

$$
\hat{f} t_{j}(\omega)=K_{j}(\omega) \cdot\left[x_{j s}(\omega)-x_{j r}(\omega)\right]
$$

where:

$\hat{f} t_{j}(\omega)$ is the operational force transmitted to path $j$, estimated by the CSM;

$K_{j}(\omega)$ is the complex stiffness of the mount for path $j$;

$x_{j s}(\omega)$ is the mount displacement for path $j$ on the source side;

$x_{j r}(\omega)$ is the mount displacement for path $j$ on the receiver side.

The second method studied is the Matrix Inversion Method (MIM). This technique was developed to be used when the stiffness is high at the attachment points of the source to the receiver structure, leading to small relative displacements and inaccuracy in the force estimation by the CSM.

The MIM is based on the so-called accelerance FRF matrix (accelerations divided by forces). To use this technique it is necessary to obtain the operational accelerations and the accelerance FRF for the attachment points, on the receiver side. Once the accelerance matrix has been determined, the equivalent forces on each path are estimated by the product between the generalized inverse accelerance matrix and the acceleration vector. The use of the singular value decomposition method helps to minimize numerical problems in the matrix inversion. The inversion of ill-conditioned matrices is discussed in [2,3]. Mathematically: 


$$
\left\{\begin{array}{l}
\hat{f}_{1} \\
\vdots \\
\hat{f}_{n}
\end{array}\right\}=\left[\begin{array}{lll}
H a_{11} & \cdots & H a_{1 n} \\
\vdots & \ddots & \vdots \\
H a_{m 1} & \cdots & H a_{m n}
\end{array}\right]^{+}\left\{\begin{array}{l}
a_{1 r} \\
\vdots \\
a_{m r}
\end{array}\right\}
$$

where:

$\left\{\begin{array}{l}a_{1 r} \\ \vdots \\ a_{m r}\end{array}\right\}$ is the operational acceleration vector on the receiver side;

$H a_{i j}$ is the accelerance FRF measured between the $\mathrm{i}^{\text {th }}$ acceleration and an equivalent force $\hat{f}_{j}$ applied to the transfer path $j$;

$\hat{f}_{i}$ is the equivalent force applied to the $\mathrm{i}^{\text {th }}$ path, estimated by the MIM;

[]$^{+}$is the generalized inverse matrix in the least-squares sense.

To obtain a unique solution to the operational forces, the number of measuring points (m) has to be at least equal to the number of forces to be estimated (n). However, redundancy of information, that is, additional response measurements $(\mathrm{m}>\mathrm{n})$ on the receiver side, tends to improve the results. This leads to a non-squared matrix to be inverted and the need of generalized matrix inversion.

\section{Techniques to obtain the frequency response functions}

The FRF are of key importance to Transfer Path Analysis. They can be obtained either analytically, from a finite element model, or experimentally. Experimental techniques to evaluate the FRF are similar to those used in modal analysis. An impact hammer or a small electromagnetic shaker can be used to excite the structure with force transducers to measure the input forces. The response can be measured on the receiver side either with accelerometers, to obtain accelerance FRF, with microphones to have the acoustic FRF (sound pressures divided by forces), or with optical methods such as laser vibrometry, to obtain velocity or displacement. According to [1], reciprocity techniques can also be used to estimate FRF with a loudspeaker placed on the receiver location and accelerometers on the source side.

Basically, two setups can be considered to estimate the FRF. The first one is the complete system, i.e., the source and the receiver connected to each other. The second one, based on practical considerations, is to obtain the FRF without the vibro-acoustic source (removing the engine, for instance). This technique was developed because, in most practical applications, it is difficult to access the receiver side of the attachments of the source to measure the FRF with a shaker or an impact hammer. In the present work, both cases will be discussed with their interaction with the two indirect methods to estimate the input forces.

\section{Using a lumped parameter model}

A simple 6-degree-of-freedom model has been used to compare the different techniques used to estimate the operational forces and the FRF aiming the application on Transfer Path Analysis. Different model parameters were tested and the results will be discussed. The TPA calculations will be also done with this model. Figure. 1a and $b$ show two basic configurations studied, with and without the source impedance.

In the system, mass $\mathbf{M}_{1}$ represents the engine mass (source), where an external force $f_{1}$ is applied. Mass $\mathbf{M}_{6}$ is the mass on the receiver place, where the response $x_{6}$ is taken. Between the source and the receiver there are two possible transfer paths $\mathrm{TP}_{A}$ (through masses $\mathrm{M}_{2}$ and $\mathrm{M}_{3}$ ) and $\mathrm{TP}_{B}$ (through masses $\mathrm{M}_{4}$ and $\mathrm{M}_{5}$ ).

In this TPA problem, the aim is to estimate the contribution of paths $\mathrm{A}$ and $\mathrm{B}$ to response $x_{6}$ of the receiver, for a given force $f_{1}$ applied to $\mathbf{M}_{1}$. Before applying the TPA equations and methods, it is interesting to determine the overall response level in order to have a baseline solution for further comparisons. So, considering the complete model given in Fig. 1a, the matrix equation can be written as: 


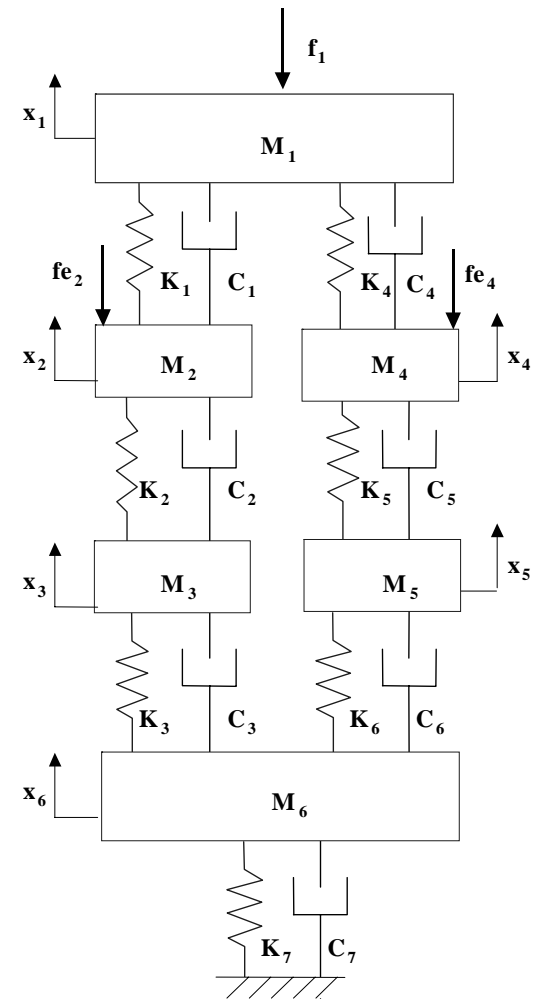

(a)

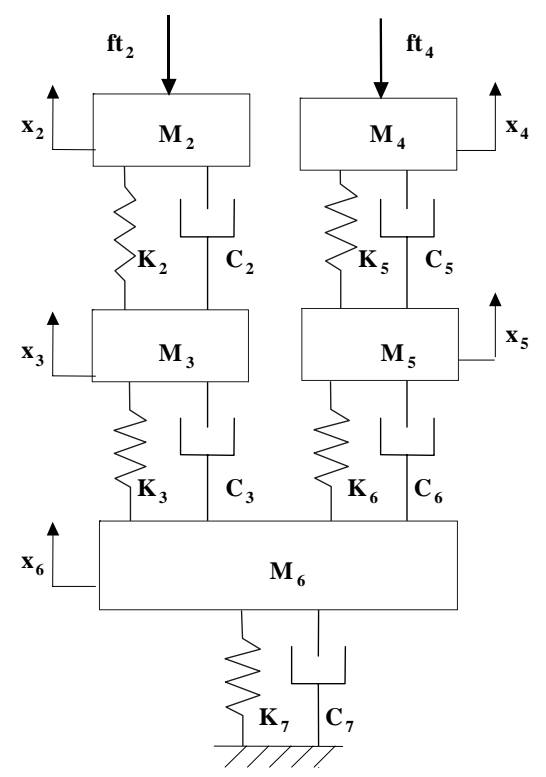

(b)

Fig. 1. (a) Model with source $M_{1}$ present; (b) model without source $M_{1}$.

$$
\left\{\begin{array}{l}
x_{1} \\
x_{2} \\
\vdots \\
x_{6}
\end{array}\right\}=\left[\begin{array}{llll}
H_{11} & H_{12} & \cdots & H_{16} \\
H_{21} & \ddots & & H_{26} \\
\vdots & & \ddots & \vdots \\
H_{61} & \cdots & \cdots & H_{66}
\end{array}\right]\left\{\begin{array}{l}
f_{1} \\
0 \\
\vdots \\
0
\end{array}\right\}
$$

Solving Eq. (4) for $x_{6}$ leads to:

$$
x_{6}(\omega)=H_{61}(\omega) \cdot f_{1}(\omega)
$$

\section{Comparison of methods}

To compare the different possibilities for applying TPA, let us first define $\tilde{H}_{i j}$ such as:

$$
\tilde{H}_{i j}=H_{i j} \text { (when the mass } \mathbf{M}_{1} \text { is present) and } \tilde{H}_{i j}=\bar{H}_{i j} \text { (when the mass } \mathbf{M}_{1} \text { is missing) }
$$

Considering the CSM, the forces $\hat{f} t_{2}$ and $\hat{f} t_{4}$ can be estimated using Eq. (2):

$$
\begin{aligned}
& \hat{f} t_{2}(\omega)=K_{1} \cdot\left[x_{1}(\omega)-x_{2}(\omega)\right]+i \omega C_{1} \cdot\left[x_{1}(\omega)-x_{2}(\omega)\right] \\
& \hat{f} t_{4}(\omega)=K_{4} \cdot\left[x_{1}(\omega)-x_{4}(\omega)\right]+i \omega C_{4} \cdot\left[x_{1}(\omega)-x_{4}(\omega)\right]
\end{aligned}
$$

For CSM, Eq. (4) becomes: 


$$
\left\{\begin{array}{l}
x_{2} \\
x_{3} \\
\vdots \\
x_{6}
\end{array}\right\}=\left[\begin{array}{ll}
\tilde{H}_{22} & \tilde{H}_{24} \\
\tilde{H}_{32} & \tilde{H}_{34} \\
\vdots & \vdots \\
\tilde{H}_{62} & \tilde{H}_{64}
\end{array}\right]\left\{\begin{array}{l}
\hat{f} t_{2} \\
\hat{f} t_{4}
\end{array}\right\}
$$

From this equation, the response $x_{6}$ can be written as:

$$
x_{6}(\omega)=\tilde{H}_{62}(\omega) \cdot \hat{f} t_{2}(\omega)+\tilde{H}_{64}(\omega) \cdot \hat{f} t_{4}(\omega)
$$

From Eq. (9), the response $x_{6}$ is written as a superposition of responses due to paths A e B. These partial contributions, according to Eq. (1), can be written as the product of the FRF by the force transmitted to each path. The force $\hat{f} t_{2}$ is transmitted to the mass $\mathbf{M}_{2}$ (transfer path $\mathrm{A}$ ) and the force $\hat{f} t_{4}$ is transmitted to $\mathbf{M}_{4}$ (transfer path B). So one can state that:

$$
x_{6}(\omega)=T P_{A}+T P_{B}
$$

where:

$$
\begin{aligned}
& T P_{A}=\tilde{H}_{62}(\omega) \cdot \hat{f} t_{2}(\omega) \\
& T P_{B}=\tilde{H}_{64}(\omega) \cdot \hat{f} t_{4}(\omega)
\end{aligned}
$$

Considering the MIM, the operational forces are obtained from the inversion of the accelerance FRF matrix according to Eq. (3). The forces can be estimated by:

$$
\left\{\begin{array}{l}
\hat{f}_{2} \\
\hat{f}_{4}
\end{array}\right\}=\left[\begin{array}{ll}
\tilde{H} a_{22} & \tilde{H} a_{24} \\
\tilde{H} a_{32} & \tilde{H} a_{34} \\
\tilde{H} a_{42} & \tilde{H} a_{44} \\
\tilde{H} a_{52} & \tilde{H} a_{54}
\end{array}\right]^{+}\left\{\begin{array}{l}
a_{2} \\
a_{3} \\
a_{4} \\
a_{5}
\end{array}\right\}
$$

where:

$\widehat{f}_{\tilde{H}^{2}}=\widehat{f} e_{2}$ and $\widehat{f}_{4}=\widehat{f} e_{4}\left(\right.$ with $\mathbf{M}_{1}$ ) and $\widehat{f}_{2}=\widehat{f} t_{2}$ and $\widehat{f}_{4}=\widehat{f} t_{4}$ (without $\mathbf{M}_{1}$ )

$\tilde{H} a_{i j}=H a_{i j}\left(\right.$ with $\mathrm{M}_{1}$ ) and $\tilde{H} a_{i j}=\bar{H} a_{i j}$ (without $\mathrm{M}_{1}$ )

So, the system can be solved using the matrix equation:

$$
\left\{\begin{array}{l}
x_{2} \\
x_{3} \\
\vdots \\
x_{6}
\end{array}\right\}=\left[\begin{array}{ll}
\tilde{H}_{22} & \tilde{H}_{24} \\
\tilde{H}_{32} & \tilde{H}_{34} \\
\vdots & \vdots \\
\tilde{H}_{62} & \tilde{H}_{64}
\end{array}\right]\left\{\begin{array}{l}
\hat{f}_{2} \\
\hat{f}_{4}
\end{array}\right\}
$$

Leading to the following equation to obtain the response $x_{6}$ :

$$
x_{6}(\omega)=\tilde{H}_{62}(\omega) \cdot \hat{f}_{2}(\omega)+\tilde{H}_{64}(\omega) \cdot \hat{f}_{4}(\omega)
$$

Similarly to Eq. (9), Eq. (15) is the TPA calculation based on the MIM. This two-component equation indicates the contribution of transfer path $\mathrm{A}$ and $\mathrm{B}$. The response $x_{6}$ can be written as:

$$
x_{6}(\omega)=T P_{A}+T P_{B}
$$

Where:

$$
\begin{aligned}
& T P_{A}=\tilde{H}_{62}(\omega) \cdot \hat{f}_{2}(\omega) \\
& T P_{B}=\tilde{H}_{64}(\omega) \cdot \hat{f}_{4}(\omega)
\end{aligned}
$$

Table 1 lists the comparison of the different methods. The FRF estimated with and without $\mathrm{M}_{1}$, respectively $H_{i j}$ and $\bar{H}_{i j}$, can be used with the two techniques to estimate the operational forces, CSM and MIM. The Matrix 
Table 1

Investigated cases

\begin{tabular}{clc}
\hline Case & Operational Force Estimation & FRF Estimation \\
\hline I & Complex Stiffness Method & $H_{i j}$ \\
\hline II & Complex Stiffness Method & $\bar{H}_{i j}$ \\
III & Matrix Inversion Method (with $H a_{i j}$ ) & $H_{i j}$ \\
IV & Matrix Inversion Method (with $\bar{H} a_{i j}$ ) & $\bar{H}_{i j}$ \\
V & Matrix Inversion Method (with $H a_{i j}$ ) & $\bar{H}_{i j}$ \\
VI & Matrix Inversion Method (with $\bar{H} a_{i j}$ ) & $H_{i j}$ \\
\hline
\end{tabular}

Inversion Method still has two possibilities for estimating the operational forces, either using the accelerance matrix with $\mathrm{M}_{1}$ or without, respectively $H a_{i j}$ and $\bar{H} a_{i j}$. So, the total number of combinations is 6 .

From the cases proposed in Table 1, cases V and VI can be discarded since they are incompatible for practical reasons. It is not reasonable, although theoretically possible, to perform a TPA by estimating the force in the presence of $\mathrm{M}_{1}$ (using $H a_{i j}$ ) and the FRF in its absence (using $\bar{H}_{i j}$ ) and vice versa. So, the comparison of the methods will be performed for the remaining four cases.

In order to test the techniques described above, the following values were assumed for the system shown in Fig. 1a:

$\begin{array}{lll}\mathrm{M}_{1}=100 \mathrm{~kg} & \mathrm{~K}_{1}=40 \mathrm{kN} / \mathrm{m} & \mathrm{C}_{1}=40 \mathrm{Ns} / \mathrm{m} \\ \mathrm{M}_{2}=10 \mathrm{~kg} & \mathrm{~K}_{2}=10000 \mathrm{kN} / \mathrm{m} & \mathrm{C}_{2}=10 \mathrm{Ns} / \mathrm{m} \\ \mathrm{M}_{3}=30 \mathrm{~kg} & \mathrm{~K}_{3}=10000 \mathrm{kN} / \mathrm{m} & \mathrm{C}_{3}=20 \mathrm{Ns} / \mathrm{m} \\ \mathrm{M}_{4}=50 \mathrm{~kg} & \mathrm{~K}_{4}=450 \mathrm{kN} / \mathrm{m} & \mathrm{C}_{4}=100 \mathrm{Ns} / \mathrm{m} \\ \mathrm{M}_{5}=50 \mathrm{~kg} & \mathrm{~K}_{5}=10000 \mathrm{kN} / \mathrm{m} & \mathrm{C}_{5}=25 \mathrm{Ns} / \mathrm{m} \\ \mathrm{M}_{6}=200 \mathrm{~kg} & \mathrm{~K}_{6}=5000 \mathrm{kN} / \mathrm{m} & \mathrm{C}_{6}=25 \mathrm{Ns} / \mathrm{m} \\ & \mathrm{K}_{7}=50 \mathrm{kN} / \mathrm{m} & \mathrm{C}_{7}=40 \mathrm{Ns} / \mathrm{m}\end{array}$

To compare the different cases, the exact solution of the complete system was used as baseline, considering the displacements $x_{1}, x_{2}, x_{3}, x_{4}$ and $x_{5}$ for the force $f_{1}$. The force $f_{1}$, applied to $\mathbf{M}_{1}$, was assumed to be constant in the frequency range from 0 to $500 \mathrm{~Hz}$ with a magnitude of $10 \mathrm{~N}$. A MATLAB code was written to run the different cases listed in Table 1.

Figures 2 and 3 indicate, respectively, the results obtained with the MATLAB code for the Complex Stiffness and the Matrix Inversion Method. Figure 2 indicates that, when CSM is used with the source removed from the system, the exact solution is achieved, coinciding with the baseline. The presence of the source $\left(\mathrm{M}_{1}\right)$ leads to a slightly different result at low frequencies. Figure 3 shows that, in MIM, the best results are obtained with the source present. A poor estimation is observed when the source is removed.

So, in the CSM, one can conclude that the estimated forces $\hat{f} t_{2}$ and $\hat{f} t_{4}$ represent exactly the transmitted forces to the paths $\mathrm{TP}_{A}$ and $\mathrm{TP}_{B}$. That is equivalent to a system without the mass $\mathrm{M}_{1}$ and with external forces $\hat{f} t_{2}$ and $\hat{f} t_{4}$ acting on the masses $\mathbf{M}_{2}$ and $\mathbf{M}_{4}$. Thus, using the $\mathbf{C S M}$, the exact solution is obtained when $\tilde{H}_{62}$ and $\tilde{H}_{64}$ are calculated without the source mass $\mathbf{M}_{1}$. In other words, the presence of $\mathbf{M}_{1}$ implies in considering the mechanical impedance of the source in the estimation of the FRF, although this impedance was already taken into account when the transmitted forces were estimated.

In the calculation using MIM, from Eq. (13), one can verify that the transmitted forces depend on the FRF matrix, $\left[\tilde{H} a_{i j}\right]$. Additionally, the calculation of the transfer paths themselves is also dependent upon the FRF matrix, [ $\left.\tilde{H}_{i j}\right]$, according to Eqs (14) and (15). Therefore, the calculation is more accurate with the complete system, i.e., with the source impedance included. In the absence of the source, both forces $\hat{f}_{j}$ and FRF $\tilde{H}_{i j}$ in Eq. (15) are not estimated properly, affecting the final calculation of the transfer paths.

The conclusion is that case II (source absent) for the Complex Stiffness Method and case III (source present) for the Matrix Inversion Method lead to the best results in the TPA calculation.

\section{Consideration on the source-receiver impedance matching}

Mechanical impedance is defined as the complex ratio of force to velocity. See [4]. In the lumped system indicated in Fig. 1, the mechanical impedance of the source will be directly proportional to the source mass $\mathbf{M}_{1}$, to the stiffness 
Table 2

Comparison of the estimation techniques with the impedance matching

\begin{tabular}{|c|c|c|c|c|c|}
\hline \multirow[t]{2}{*}{ Case } & \multicolumn{2}{|c|}{ Estimation Technique } & \multicolumn{3}{|c|}{ Impedance Matching } \\
\hline & Operational Force & FRF & Low $\mathrm{K}_{1}$ and $\mathrm{K}_{4}$ & Medium $\mathrm{K}_{1}$ and $\mathrm{K}_{4}$ & High $\mathrm{K}_{1}$ and $\mathrm{K}_{4}$ \\
\hline I & CSM & $H_{i j}$ & good & fair & poor \\
\hline II & CSM & $\bar{H}_{i j}$ & good & good & good \\
\hline III & MIM (with $H a_{i j}$ ) & $H_{i j}$ & good & good & good \\
\hline IV & MIM (with $\bar{H} a_{i j}$ ) & $\bar{H}_{i j}$ & poor & poor & poor \\
\hline
\end{tabular}

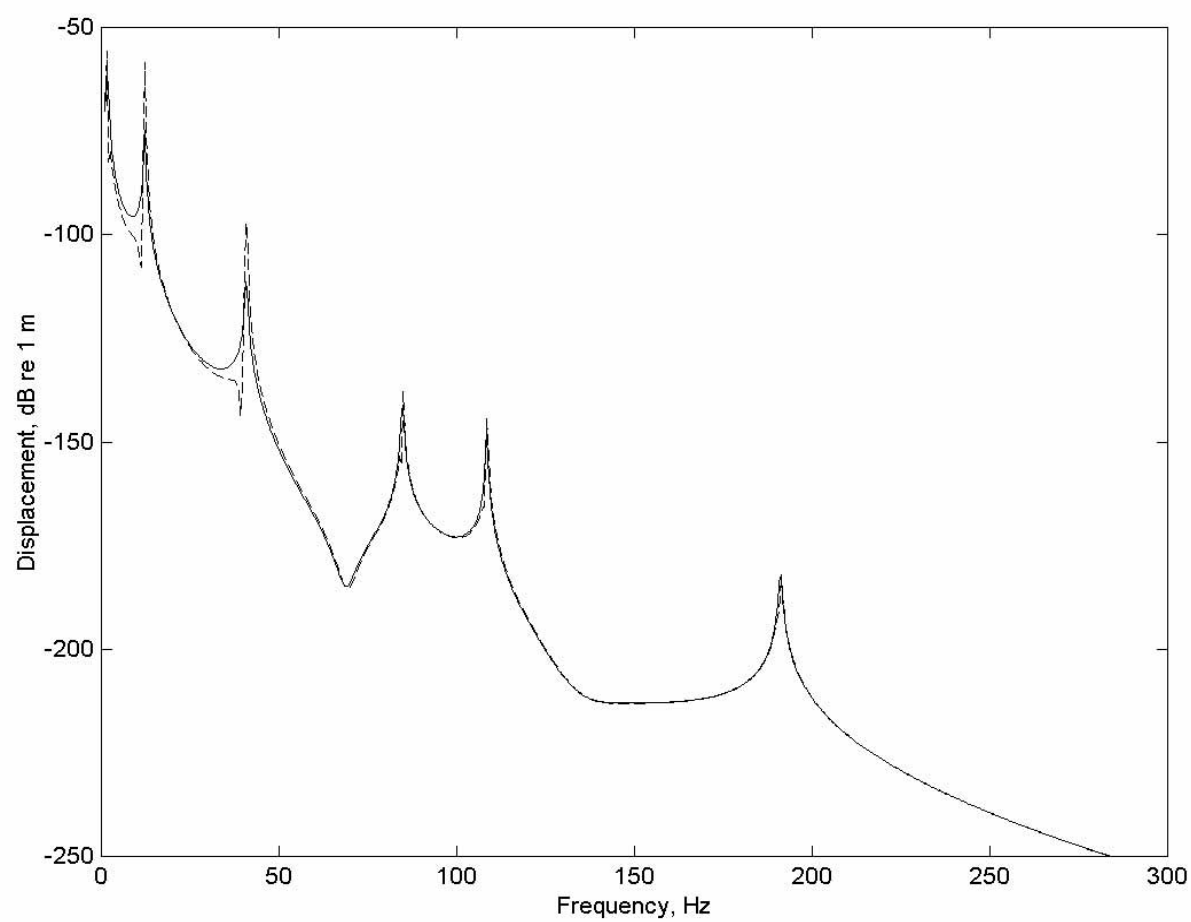

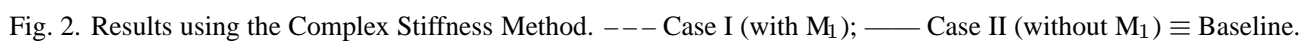

coefficients $\mathrm{K}_{1}$ and $\mathrm{K}_{4}$ and to the damping coefficients $\mathrm{C}_{1}$ and $\mathrm{C}_{4}$. The stiffness and damping coefficients form the interface between the source and the receiver. Changing these parameters will affect the so-called impedance matching between the source and the receiver. In the present case, impedance mismatch will occur when these parameters are set to very small values and good matching will be achieved with high values of them.

To check how the impedance match/mismatch can affect the estimation techniques listed in Table 1, the MATLAB program was run with stiffness $\mathrm{K}_{1}$ varying from 0.004 to $400,000 \mathrm{kN} / \mathrm{m}$, and $\mathrm{K}_{4}$ varying from 0.045 to $4,500,000 \mathrm{kN} / \mathrm{m}$, for cases I to IV. The comparison of the results obtained with the baseline is listed in Table 2.

According to Table 2, one can infer that the best estimation techniques previously discussed, cases II and III, are independent of the impedance matching between the source and the receiver. This means that both cases are not affected by the interface parameter values and can be applied to any attachment condition, i.e., from rigid to resilient connections between the source and the receiver. Case IV gives poor results for any attachment condition and should be discarded. Case I leads to good results only if there is a reasonably high impedance mismatch between the source and the receiver and can be used only when this condition is satisfied. 


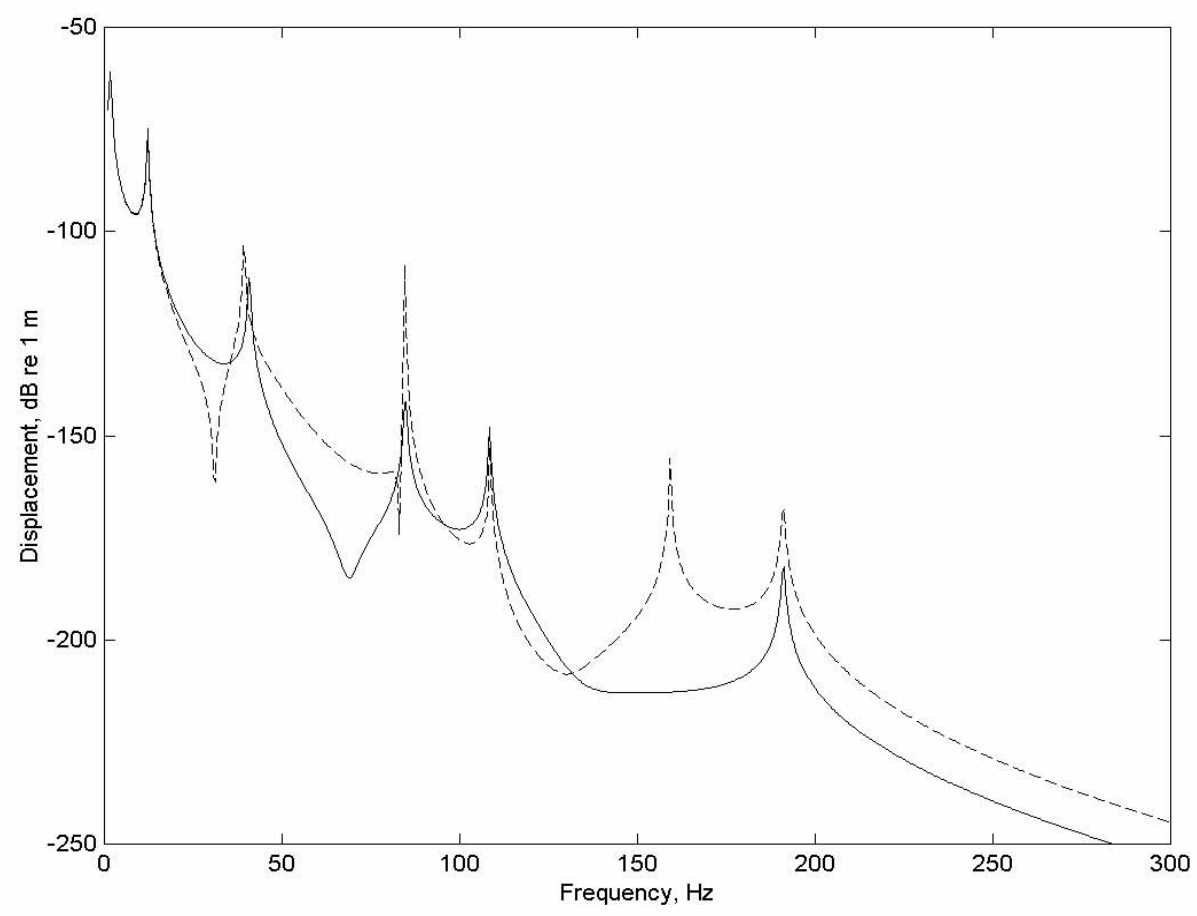

Fig. 3. Results using the Matrix Inversion Method. —_ Case III (with $\mathrm{M}_{1}$ ) $\equiv$ Baseline; --- Case IV (without $\mathrm{M}_{1}$ ).

\section{The effect of coupling between paths}

In the simplified model used to test the different techniques, no coupling was assumed between the two transfer paths. In practical situations however, coupling can occur among paths and the energy can flow from one path to another before the energy reaches the receiver. The best techniques (cases II and III) were tested for different couplings between the two paths. Starting from the same model shown in Fig. 1, a spring and a damper are attached between M3 and M5. Varying $\mathrm{K}$ and $\mathrm{C}$ of this attachment from zero to higher values, the effect of weak and strong coupling between the two paths was simulated. The MATLAB code was adapted to run these cases.

The conclusion is that the results are independent of the coupling between paths for both methods.

\section{Concluding remarks}

When applying TPA, forces and FRF should be measured. The direct measure of exciting forces is not possible in most cases and indirect methods to estimate the forces should be used instead. Two methods were presented in this paper, the Complex Stiffness Method and the Matrix Inversion Method.

With the source (e.g. the vehicle engine) removed, CSM gives more accurate results. On the other hand, with the source installed, MIM is preferable. In the latter case, CSM can be only used if there is a high impedance mismatch between the source and the receiver. Both methods are not affected by a higher or lower degree of coupling among the transfer paths.

\section{References}

[1] LMS International, Transfer Path Analysis: The Qualification and Quantification of Vibro-acoustic Transfer Path, Leuven, Belgium, 2000. 
[2] A.N. Thite and D.J. Thompson, The Quantification of Structure-borne Transmission Paths by Inverse Methods. Part 1: Improved Singular Value Rejection Methods, Journal of Sound and Vibration 264 (2003), 411-431.

[3] A.N. Thite and D.J. Thompson, The Quantification of Structure-borne Transmission Paths by Inverse Methods. Part 2: Use of Regularization Techniques, Journal of Sound and Vibration 264 (2003), 433-451.

[4] P. Hynnä, Mechanical Mobility Technique, VTT Technical Research Centre of Finland, Research Report No. BVAL37-021228, 2002. 

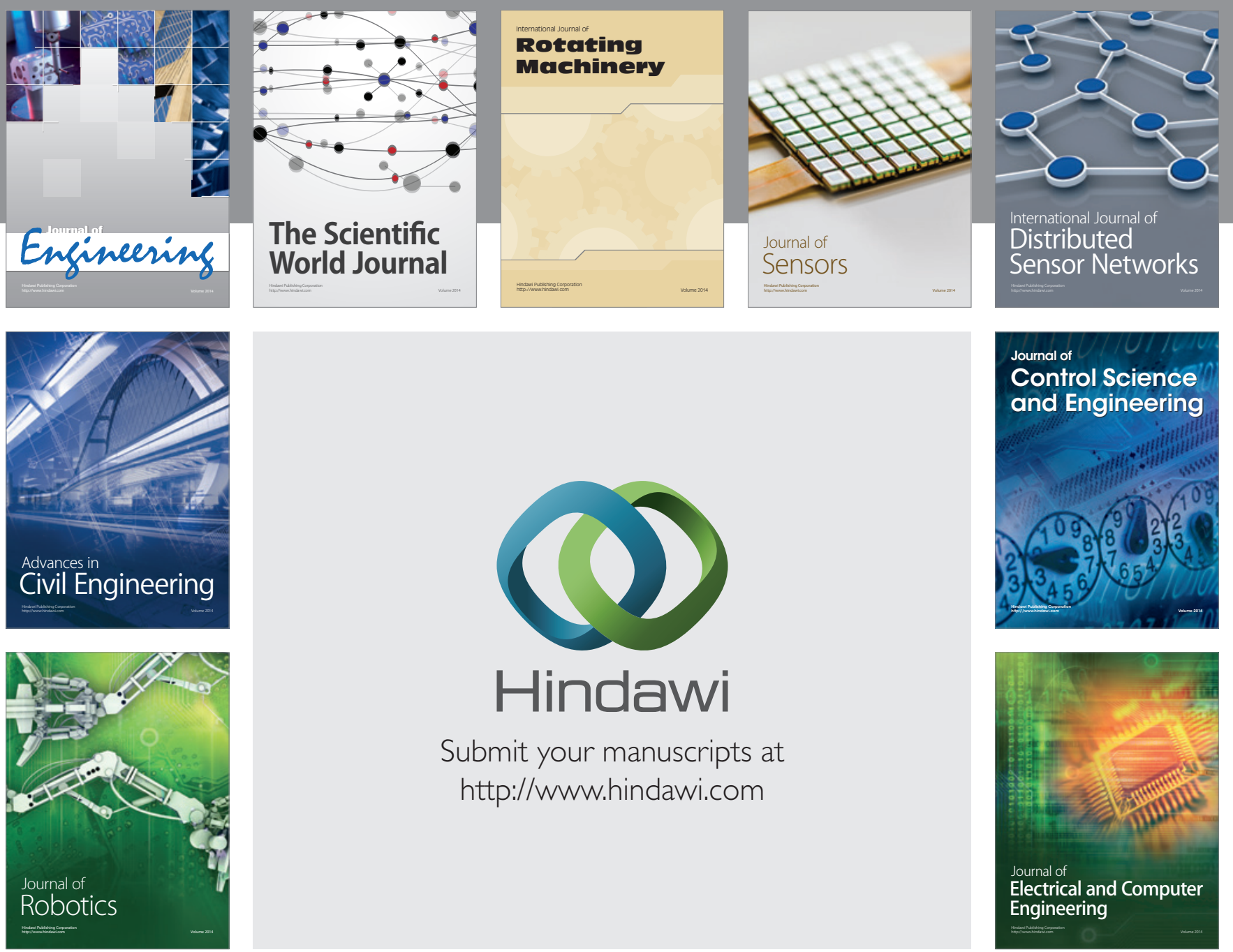

Submit your manuscripts at

http://www.hindawi.com
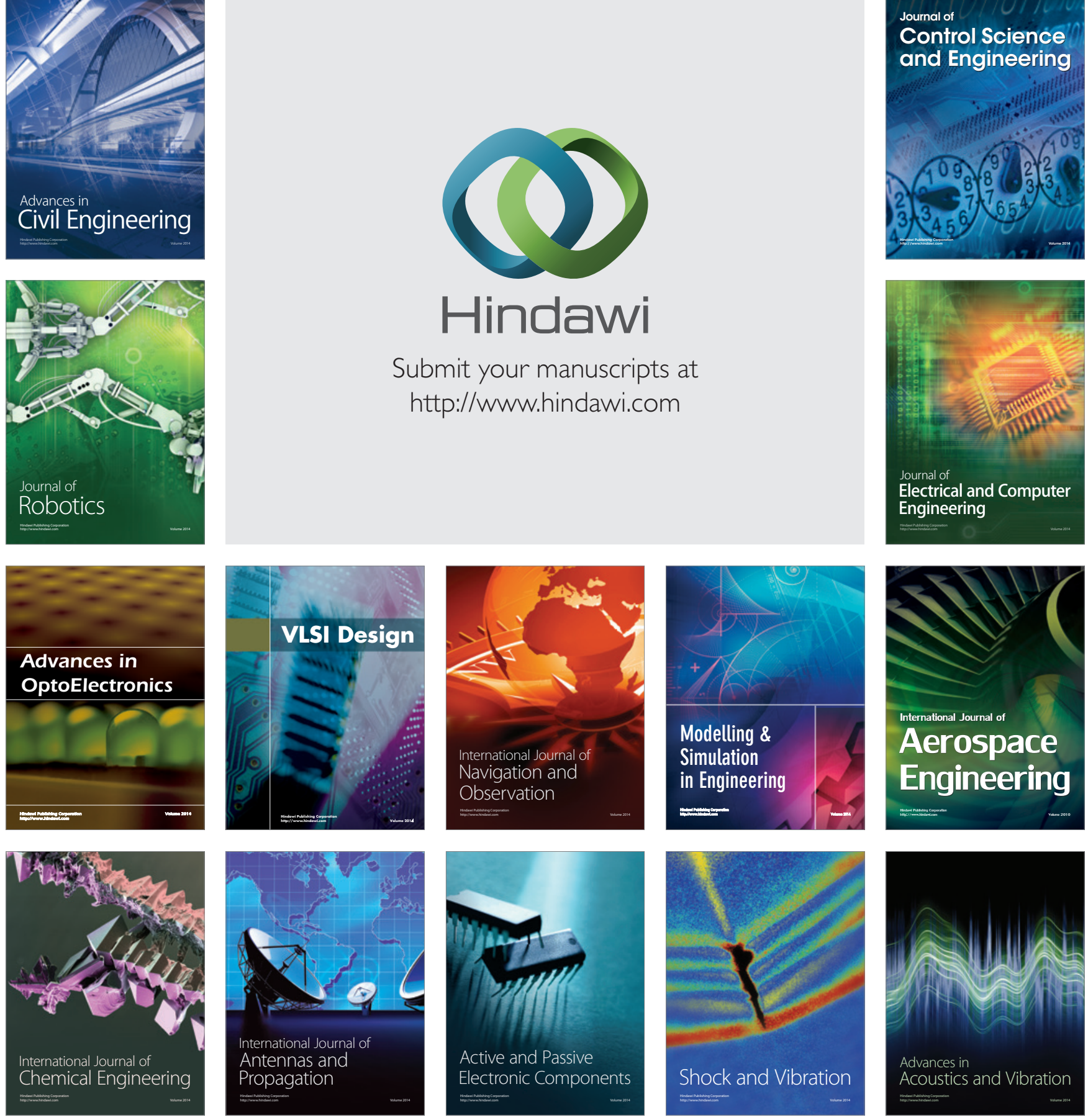